\title{
A Self-Healing Liquid Metal Anode with PEO-Based Polymer Electrolytes for Rechargeable Lithium Batteries
}

Tianyi $\mathrm{Li}^{1,2 \ddagger}$, Yi Cui ${ }^{1,2 \ddagger}$, Longlong Fan $^{3}$, Xinwei Zhou ${ }^{1,2}$, Yang Ren ${ }^{3}$, Vincent De Andrade $^{3}$, Francesco De Carlo ${ }^{3}$ and Likun Zhu ${ }^{1 *}$

${ }^{1}$ Department of Mechanical and Energy Engineering, Indiana University-Purdue University Indianapolis, Indianapolis, IN 46202, United States

${ }^{2}$ School of Mechanical Engineering, Purdue University, West Lafayette, IN 47907, United States

${ }^{3}$ Advanced Photon Source, Argonne National Laboratory, Argonne, IL 60439, United States

${ }^{*}$ Corresponding author: likzhu@iupui.edu (L. Zhu)

$\$$ These authors contributed equally to this work

This is the author's manuscript of the article published in final edited form as: 


\section{Abstract}

In this study, a Ga-Sn liquid metal alloy material is demonstrated as a self-healing anode system due to its fluidity via operando synchrotron-based transmission X-ray microscopy and X-ray diffraction experiments. Cracks formed due to volume expansions can be recovered by the fluidity of the liquid metals. By incorporating with a poly(ethylene oxide) (PEO)-based electrolyte at $60^{\circ} \mathrm{C}$, the $\mathrm{Ga}-\mathrm{Sn}$ anode shows a reversible lithium insertion and extraction process with a high initial discharge specific capacity of $682 \mathrm{mAh} \mathrm{g}^{-1}$, followed by delivering a capacity of $462 \mathrm{mAh} \mathrm{g}^{-1}$ in the second cycle at $\mathrm{C} / 20$ rate. Compared with its solid counterparts, the Ga-Sn liquid metal anode demonstrates a better capability to maintain its mechanical integrity and better contact with PEO solid electrolytes due to its advantageous features of the liquid. This study suggests a potential strategy to use liquid metal alloys with polymer solid electrolyte to solve the anode challenges in rechargeable lithium batteries.

\section{Graphical abstract}

The gallium tin liquid metal anode coupled with poly(ethylene oxide) solid electrolyte tested at $60{ }^{\circ} \mathrm{C}$ demonstrate better mechanical stability and reversible electrochemical reactions due to its self-healing characteristics, therefore, provides a potential scheme to employ liquid metal alloys with polymer-based solid electrolyte to accommodate the anode difficulties in rechargeable lithium ion batteries. 

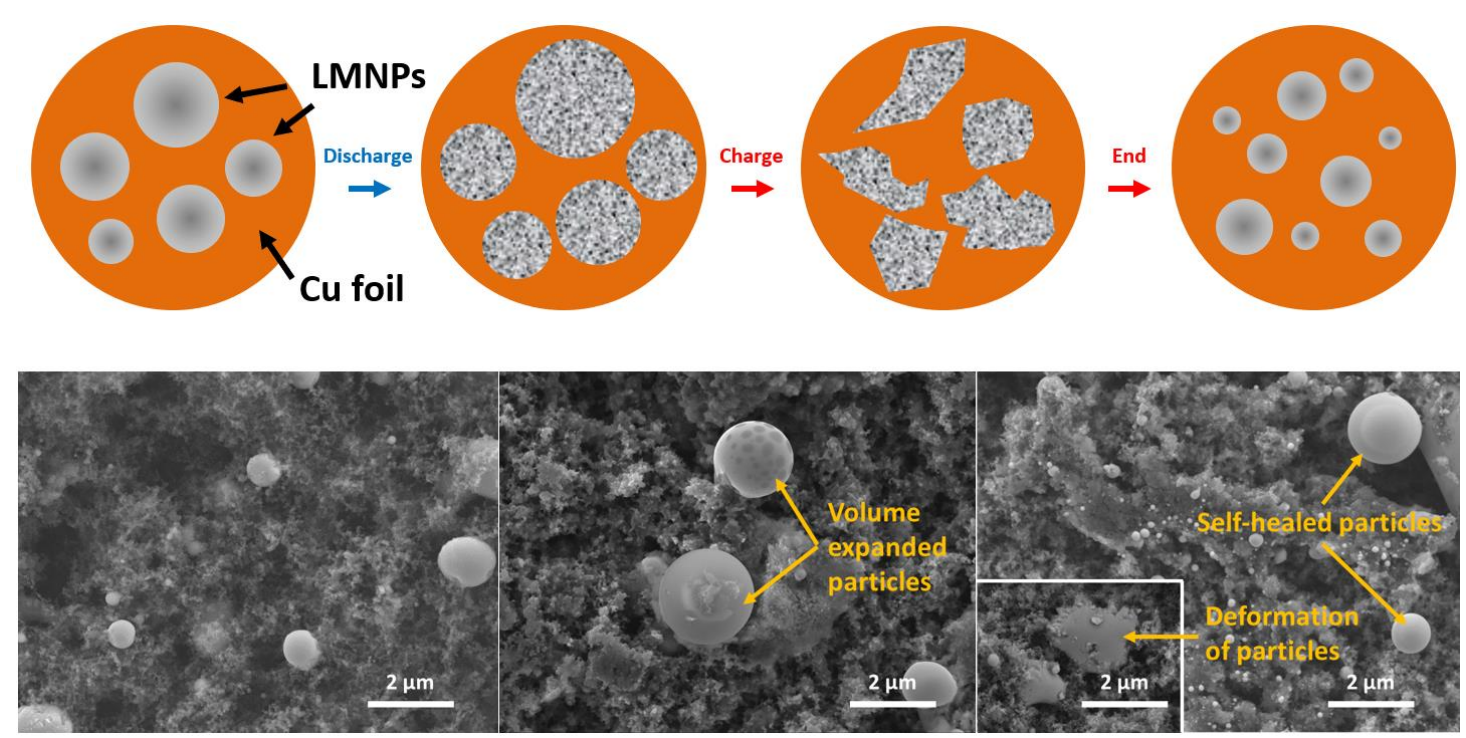

\section{Keywords}

Lithium ion batteries, Self-healing anode, Gallium tin alloy, Polymer solid electrolyte

\section{Highlights}

- The concept of using Ga-Sn liquid metal as low melting point anode materials for lithium ion batteries is presented.

- The chemical and crystalline evolutions were validated via transmission X-ray microscopy and X-ray diffraction.

- The polymer-based solid electrolyte was employed leads to better mechanical integrity due to the self-healing ability of Ga-Sn alloy at room temperature. 


\section{Introduction}

Energy storage has become one of the most important research areas in the $21^{\text {st }}$ century owing to fast and significant development in technology. In the past two decades, lithium-ion batteries (LIBs) have been extensively applied in portable electronic devices, electric vehicles (EVs) as well as large-scale energy storage systems favored for their high energy density. However, the graphite used as the anode in current commercial LIBs has limited specific capacity and volumetric capacity (372 $\mathrm{mAh} \mathrm{g}^{-1}$ and $837 \mathrm{mAh} \mathrm{cm}^{-3}$ ) [1]. Recently, Li-alloy materials (e.g. Si, Ge, and Sn) have attracted much attention as anode materials due to their higher theoretical specific capacity. However, Li-alloy materials have not yet been widely applied in commercial batteries because of some unresolved technical challenges [29]. The primary challenge is their unsatisfied cycling performance resulted from the large volume expansion/contraction during lithiation and delithiation reactions $[4,10-$ 12]. Such a huge volume change causes mechanical cracks of the anode particles, detachments of the active materials from the current collector, and poor contacts with the electronically conductive networks, which lead to the deactivation of active materials. Besides, the Li-alloy materials usually have insufficient Coulombic efficiency (CE) and dysphoric solid electrolyte interphase (SEI) layer during cycling.

To overcome these challenges, many ongoing efforts have been devoted to developing Li-alloy anode materials with promising cycling performance, such as intermetallic electrode [13-15], doped alloys [4, 5, 16], and nano-structuring active materials [17- 
25]. Among recent approaches, liquid metals (LM) with low melting points have been proposed as anode candidates due to their self-healing behavior and high theoretical capacity [26-28]. Gallium (Ga) is a unique metal with a low melting point $\left(29.8^{\circ} \mathrm{C}\right)$ which can deliver a theoretical capacity of $769 \mathrm{mAh} \mathrm{g}^{-1}$ by taking up to two lithium atoms for full lithiation, therefore reported as a promising LM anode material by Deshpande et al. and a few other studies [29-31]. Furthermore, Guo et al. applied GaIn alloy with a melting point as low as $15^{\circ} \mathrm{C}$ in both $\mathrm{Li}$-ion and $\mathrm{Na}$-ion batteries showing promising cyclic durability and capacity [32]. Wu et al. stabilized Ga-Sn alloy in reduced graphene oxide and carbon nanotube framework with self-healing ability and remarkable cycling performance [26]. However, the change between liquid and solid states during cycling causes continuous SEI layer growth, which leads to a gradual capacity fade. And most importantly, continuous SEI layer growth is not practical in a full cell setup with limited lithium resources. To address this issue, the electrolyte used in batteries involving LM anode should be stable on the LM surface. Solid-state electrolytes have shown much better stability than their liquid counterpart. Recently, Jin et al. have reported solid electrolyte-based molten lithium battery systems operates at $200{ }^{\circ} \mathrm{C}$ constructed with a molten lithium anode, a molten cathode, and a garnet-type $\mathrm{Li}_{6.4} \mathrm{La}_{3} \mathrm{Zr}_{1.4} \mathrm{Ta}_{0.6} \mathrm{O}_{12}$ (LLZTO) solid electrolyte tube [33, 34]. Compared to the solid-solid interface in traditional solid-state batteries, the liquid-solid interface facilitates rapid ion transport, leading to high power and long cycle life battery systems. To this end, LMs with room temperature melting point in solid electrolyte battery systems have not been widely explored. The objective of this 
paper is to investigate a liquid Ga-Sn alloy system that has a melting point around room temperature incorporated with a solid electrolyte in rechargeable lithium batteries.

In this study, Poly(ethylene oxide) (PEO) was chosen to be the solid electrolyte. The liquid Ga-Sn alloy has a weight ratio of 92:8, which yields a eutectic melting point of $25^{\circ} \mathrm{C}$. This Ga-Sn alloy has a theoretical capacity of $787 \mathrm{mAh} \mathrm{g}^{-1}$ based on the capacities of gallium and tin (993 $\left.\mathrm{mAh} \mathrm{g}^{-1}\right)$. By using PEO solid electrolyte instead of liquid electrolytes, the safety of the battery is further enhanced. In order to achieve the optimum conductivity of the PEO electrolyte, the cells were tested at $60{ }^{\circ} \mathrm{C}$. The GaSn liquid metal half cells show an initial specific capacity of $682 \mathrm{mAh} \mathrm{g}^{-1}$ and a specific capacity of $462 \mathrm{mAh} \mathrm{g}^{-1}$ in the $2^{\text {nd }}$ cycle. The morphology change of LM particles during cycling was evaluated by SEM characterizations. The crystalline and chemical evolutions were monitored via operando X-ray diffraction (XRD) and X-ray absorption near edge spectroscopy using synchrotron-based transmission X-ray microscopy with energy scan at the Advanced Photon Source (APS) at Argonne National Laboratory (ANL).

\section{Results and Discussion}

Both Ga and Sn have a high theoretical specific capacity of $769 \mathrm{mAh} \mathrm{g}^{-1}$ and 993 $\mathrm{mAh} \mathrm{g}^{-1}$, respectively. Therein, the Ga-Sn LM alloy with its weight ratio of 92:8 for Ga over Sn applied in this contribution has a calculated theoretical capacity of 787 
$\mathrm{mAh} \mathrm{g}^{-1}$, which is slightly higher than twice that of commonly used graphite anode. The Ga-Sn LM alloy has a melting point of $25^{\circ} \mathrm{C}$, which is exactly at the typical room temperature (RT). Also, it has been mentioned that the Ga-Sn LM alloy usually has a super-cooling capability for $5-6{ }^{\circ} \mathrm{C}$ below its melting point in ref [26], in which the alloy can remain as a liquid at RT conditions even though its melting point is 25 ${ }^{\circ} \mathrm{C}$. The liquid Ga-Sn LM alloy was originally placed in a coin cell case at RT, as shown in Figure 1a. It was initially a bulk liquid metal with a smooth and glittery surface because of light reflections. After discharge, the Ga-Sn alloy faded into ragged and black solid cracks due to the large volume expansion during the full lithiation process. The volume expansion is $160 \%$ and $260 \%$ for $\mathrm{Ga}$ and $\mathrm{Sn}$, respectively. Therefore, it is expected to have an approximate twice expansion for the Ga-Sn alloy after lithiation. The Ga-Sn alloy was partially recovered to smooth and glittery liquid metals after the delithiaton process with parts of the alloy remain at expanded and fractured status due to the loss of connection with PEO solid electrolyte after full lithiation, therefore, fails to release lithium during delithiation process. Using bulk Ga-Sn LM alloy will cause difficulty and deterioration for alloy recovery after lithiation. Therefore, making Ga-Sn LM alloy into Ga-Sn liquid metal nanoparticles (LMNPs) via super ultrasonication and surfactant additives helps nanoparticles maintain contact with conductive carbon and LiTFSI salt. After ultrasonication of bulk liquid metals into LMNPs, the small particles help to effectively accelerate the diffusion process and reinforce contacts as described in Figure 1b. The LMNPs expand to larger particles due to the volume expansion after lithiation. During the 
delithiation process, the particles deform into random shapes because of the large volume changes and inhomogeneity of lithium concentration inside the LMNPs.

However, after full delithiation where the condition returns to the formation of liquid metal, the particles reach liquid phases and self-heal themselves to regenerate

\section{LMNPs.}

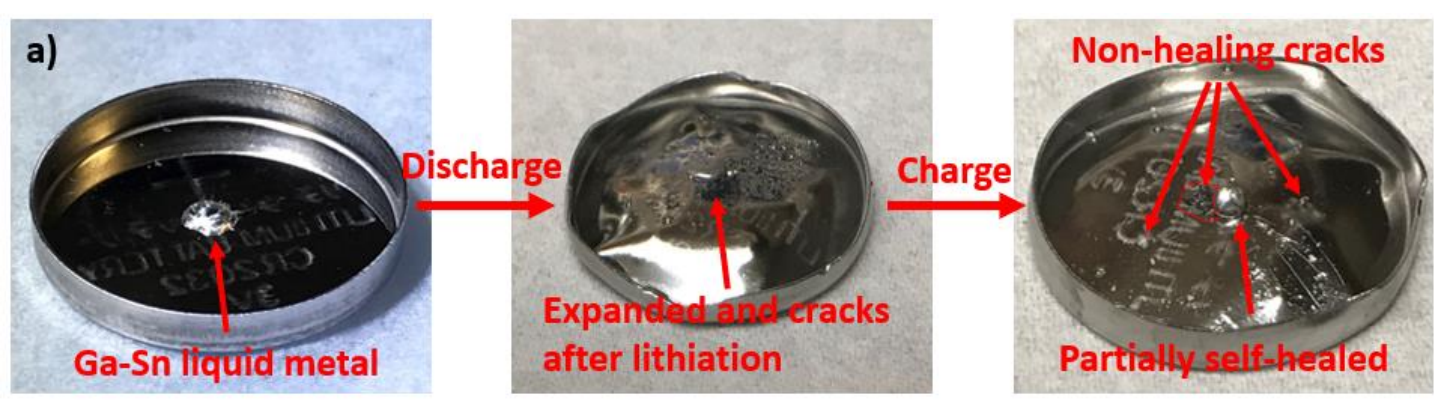

b)
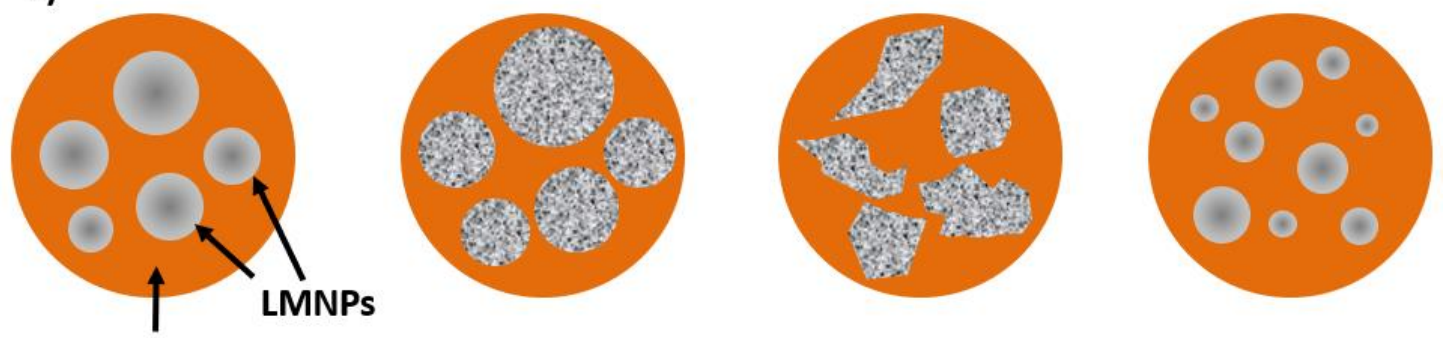

\section{Cu foil}
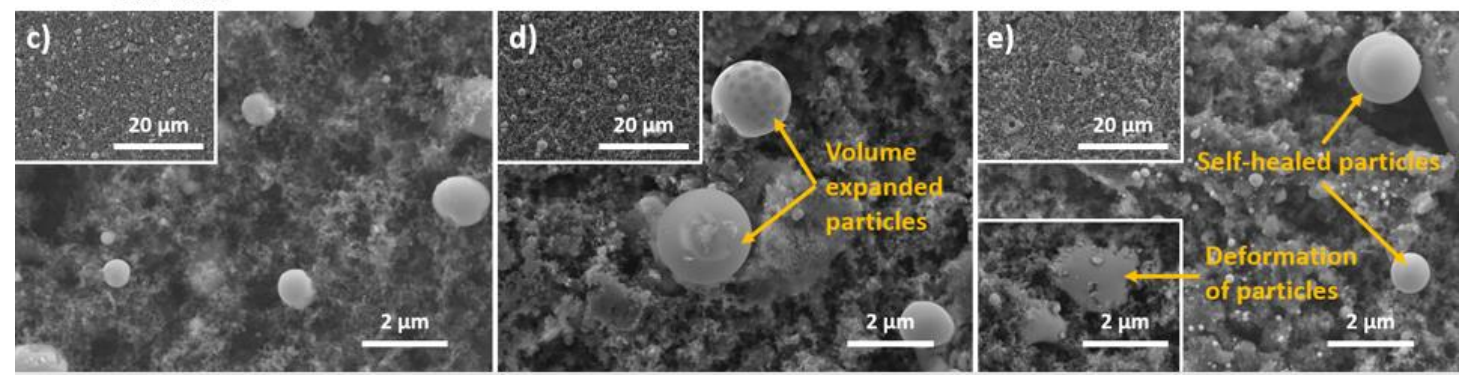

Figure 1. (a) Ga-Sn liquid metal at the pristine, discharged, charged state. (b) reaction schematics of LMNPs during lithiation and delithiation processes. (c) SEM images of (c) pristine Ga-Sn LMNPs electrode. (d) lithiated Ga-Sn LMNPs electrode. (e) delithiated Ga-Sn LMNPs electrode with a low magnified image at the upper left corner. 
The morphological changes during lithiation and delithiation were observed by SEM. The initial as-prepared LMNPs electrode was observed to have multiple nm-sized spherical particles as shown in Figure 1c with an inset image showing low magnified electrode morphology. The EDS scanning in Figure S1 shows the overlap mapping, elemental mapping of carbon, oxygen, and Ga-Sn alloy, respectively. The formation of spherical nanoparticles is due to the high surface tension of the Ga-Sn alloy droplet. The SEM image shows that the Ga-Sn particles were well connected to carbon additives, LiTFSI salt, and PEO, which provides the transport of lithium ions and electrons. After full lithiation, the LNMPs were observed to have large volume expansions by assimilating lithium into the Ga-Sn alloy lattice as shown in Figure 1d. The SEM image in Figure 1e displays the formation of smaller and glittery surfaced Ga-Sn particles after the delithiation process with some of the delithiated Ga-Sn particles occupied non-spherical shape highlighted in the bottom left image. The lower inset SEM image reveals the deformed particles could potentially due to the reduced surface tension by partially dealloying lithium ions.

To understand the lithiation mechanisms for Ga lattice in Ga-Sn alloy nanoparticles, synchrotron-based transmission X-ray microscopy (TXM) and XRD were employed under the operational condition as advanced characterizations. Because of the lack of heating capability at the synchrotron X-ray stations at the APS, 1 M LiTFSI in DME : DOL (50:50) liquid electrolyte was used for the operando XRD and TXM experiments. The voltage profile in Figure 2a shows the first cycling process at $0.1 \mathrm{C}$ 
of the LMNP electrode with red dots indicating when the TXM images were collected. Each image stack contains a series of two-dimensional transmission X-ray microscopy images as well as the energy information where each frame was collected. The intensity spectrum at each energy was normalized to generate the X-ray absorption near edge spectroscopy (XANES) datasets as shown in Figure 2b. The XANES spectrum with a white line position of $10372 \mathrm{eV}$ at the pristine state rather than $10375 \mathrm{eV}$ for gallium (III) indicates the LMNPs were gallium (O) after preparation $[35,36]$. Once the lithiation proceeded, the normalized intensity of XANES spectra decreased and became broader gradually after the voltage reaches $0.75 \mathrm{~V}$ plateau as lithium ion intercalated with a formation of $\mathrm{Li}_{2} \mathrm{Ga}$. After the voltage reaches $0.5 \mathrm{~V}$, the spectra displayed an even broader shape corresponding to the formation of the LiGa phase. After the $0.5 \mathrm{~V}$ plateau, the voltage gradually reduced to $0 \mathrm{~V}$ without a plateau for $\mathrm{Li}_{2} \mathrm{Ga}$, which indicates that $\mathrm{Li}_{2} \mathrm{Ga}$ crystal was not formed. The XANES spectrum also shows that the absorption peak continuously broadens until the end of lithiation. We believe that lithium was still inserted into $\mathrm{Li}_{\mathrm{x}} \mathrm{Ga}$ to make $\mathrm{x}$ greater than 1 . However, crystalline $\mathrm{Li}_{2} \mathrm{Ga}$ was not formed. The diminishing of the peak at $10372 \mathrm{eV}$ is an indicator of lithium ion intercalation and the formation of $\mathrm{Li}_{\mathrm{x}} \mathrm{Ga}$ type alloy. The broadening of the peak shape means that the electrons in $\mathrm{Ga}$ are less confined [37], which could be due to the amorphization process or crystal lattice increase. The spectrum after lithiation does not have a bimodal appearance demonstrates the final product is not $\mathrm{Li}_{2} \mathrm{Ga}$ yet. During the process of delithiation, the lithium ion was extracted from the system, The $\mathrm{Li}_{\mathrm{x}} \mathrm{Ga}$ 
phase was first delithiated back to $\mathrm{LiGa}$ and $\mathrm{Li}_{2} \mathrm{Ga}_{7}$ thereafter as the spectra were reinstated back to a sharper shape. The last gallium spectrum was able to recover back to its original sharp appearance at the end of the delithiation process with an identical shape and white line position as its pristine state, which indicates the chemical composition of Ga-Sn LMNPs has been fully recovered.

To investigate the morphological changes of LMNPs, image registration and subtraction were performed by using two images (one of each as shown in Figure 2cd) from the pristine and the end of delithiation. The differences between these two images (black spots in Figure 2e) indicate only a few large particles possess irregular shape after delithiation while most of the LMNPs were able to restore their original shape and positions during the cycling process, which is consistent with the SEM results shown in Figure 1. The particle size analysis based on ex-situ SEM images is shown in Figure S2. Before lithiation, the fresh Ga-Sn alloy electrode contains mostly small nm-sized particles. The nm-sized particles were initially obtained from the GaSn alloy bulk via the ultrasonication process and surfactant which ensure possible good contact with the carbon and lithium salt (LiTFSI), as well as effective lithiation reactions. The amount of nm-sized Ga-Sn particles decreases as lithiation proceeded was due to their volume expansions. Once the battery was fully charged, the population of small particles increased again. This ex-situ experiment contains discrepancies due to the variations on each different electrodes but provides some insights into morphological evolutions of the Ga-Sn system. The overall 
morphological changes indicate a promising reversible lithiation and delithiation process for the use of LMNP electrodes.
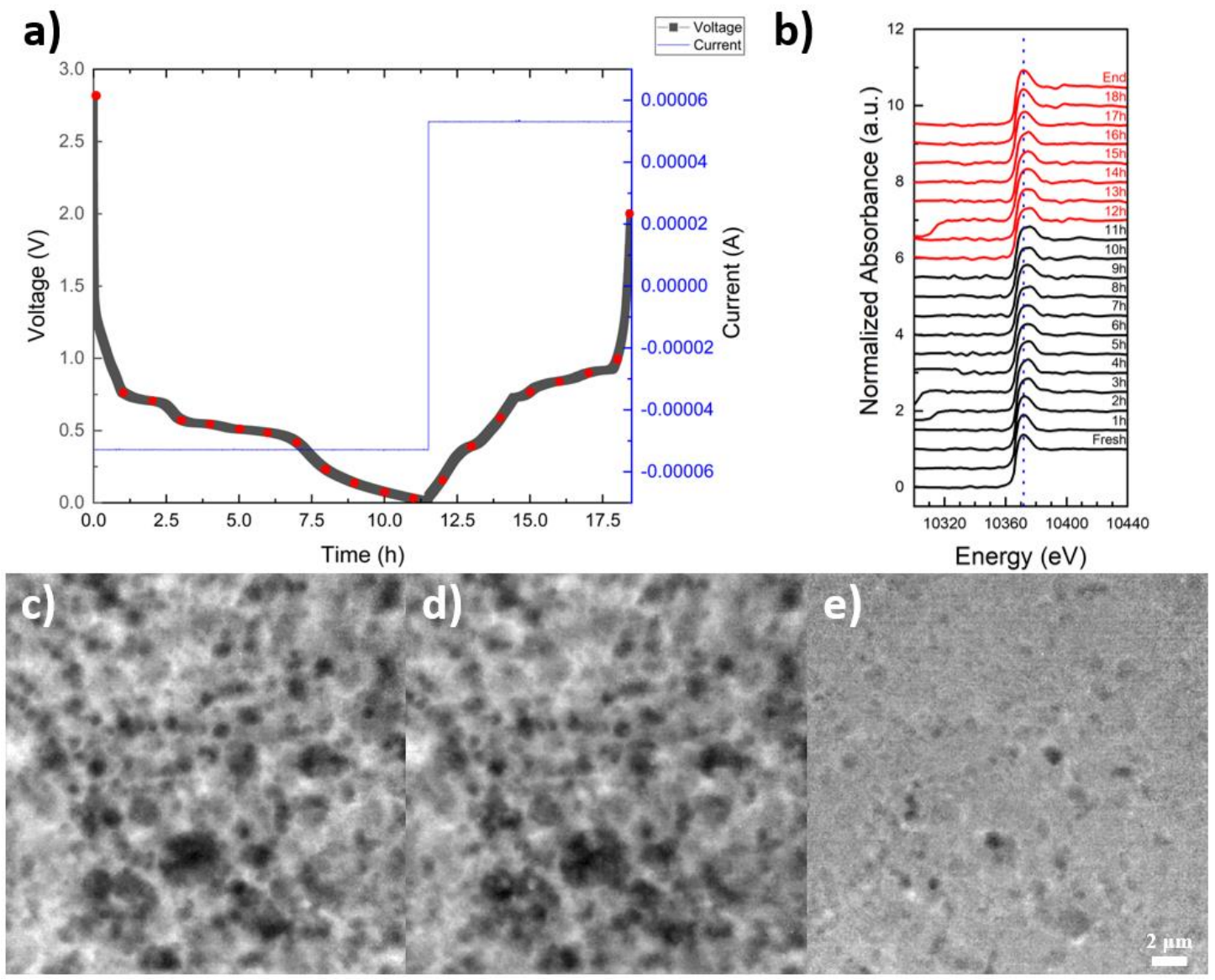

Figure 2. Operando TXM results for Ga-Sn LMNPs. (a) Voltage and current plots with highlighted data collecting points. (b) gallium XANES results during lithiation (black) and delithiation (red) processes. (c) TXM image of the pristine Ga-Sn LMNPs electrode. (d) TXM image of the delithiated Ga-Sn LMNPs electrode. (e) differences between (c) and (d) by image registration and subtraction.

To further understand the phase and morphological changes observed via the TXM experiment, an operando XRD experiment was conducted. As shown in Figure 3, the 
Ga-Sn LMNPs do not have diffraction patterns due to the nature of liquid metal at the very beginning. The LMNPs started to form $\mathrm{Li}_{2} \mathrm{Ga}_{7}$ as the lithiation process proceeded to below the plateau of $0.75 \mathrm{~V}$. Afterward, we observed the disappearance of $\mathrm{Li}_{2} \mathrm{Ga}$, and thereafter the formation of the LiGa phase at $0.5 \mathrm{~V}$. Both of these two observations were consistent with the TXM results. Instead of forming the orthorhombic $\mathrm{Li}_{2} \mathrm{Ga}$ phase at the end of the discharge process based on the calculations shown in Figure S3, we found that all the LiGa patterns shifted to smaller two theta values. This phenomenon indicates the increases of the lattice parameter of LiGa crystal during lithiation, which is also consistent with the peak broadening after the $0.5 \mathrm{~V}$ plateau in the XANES spectrum. Previous studies had demonstrated the formation of $\mathrm{Li}_{2} \mathrm{Ga}$ from $\mathrm{LiGa}$ is a structural transition from a threedimensional structure to a two-dimensional structure. This transition could cause atomic bond breakage and rearrangement which means a reaction of this kind is not likely to take place without enough kinetic conditions such as very low current and high temperature $[38,39]$. The formation of a new discrete phase yields a steady potential over the range in which one solid phase is being converted into another followed by an abrupt change in potential when the reaction is completed. In contrast, the lithiation process with a gradual change in potential from $0.5 \mathrm{~V}$ to the end of lithiation is a process of solid solution formation [38]. The operation conditions were constant current at $0.1 \mathrm{C}$ and room temperature for the TXM and XRD experiments, which cannot form the $\mathrm{Li}_{2} \mathrm{Ga}$ phase. During the process of delithiation, the extraction of lithium ions from the LiGa phase leads to the decrease of its lattice parameter that 
is demonstrated by the shifting of LiGa patterns to the larger two theta values. It is also indicated that the solid solution formation in $\mathrm{LiGa}$ crystal is reversible. $\mathrm{Li}_{2} \mathrm{Ga}_{7}$ phase was formed after the disappearance of LiGa and vanished at the end of delithiation. The operando XRD of the second cycle behaves identically as the first cycling performance. There was no observation of any $\mathrm{Li}_{\mathrm{x}} \mathrm{Sn}$ phases among its first two cycles implies either the small portion of Sn is below the detecting limit or the main effect of $8 \% \mathrm{Sn}$ is to reduce the melting point of LMNPs without influencing the crystal structures of $\mathrm{Ga}$ or participating in the electrochemical reaction during cycling. For verification, we have also conducted another operando XRD experiment by employing pure Ga LMNPs under the same operational condition showing the same result as displayed in Figure S4. 
a)

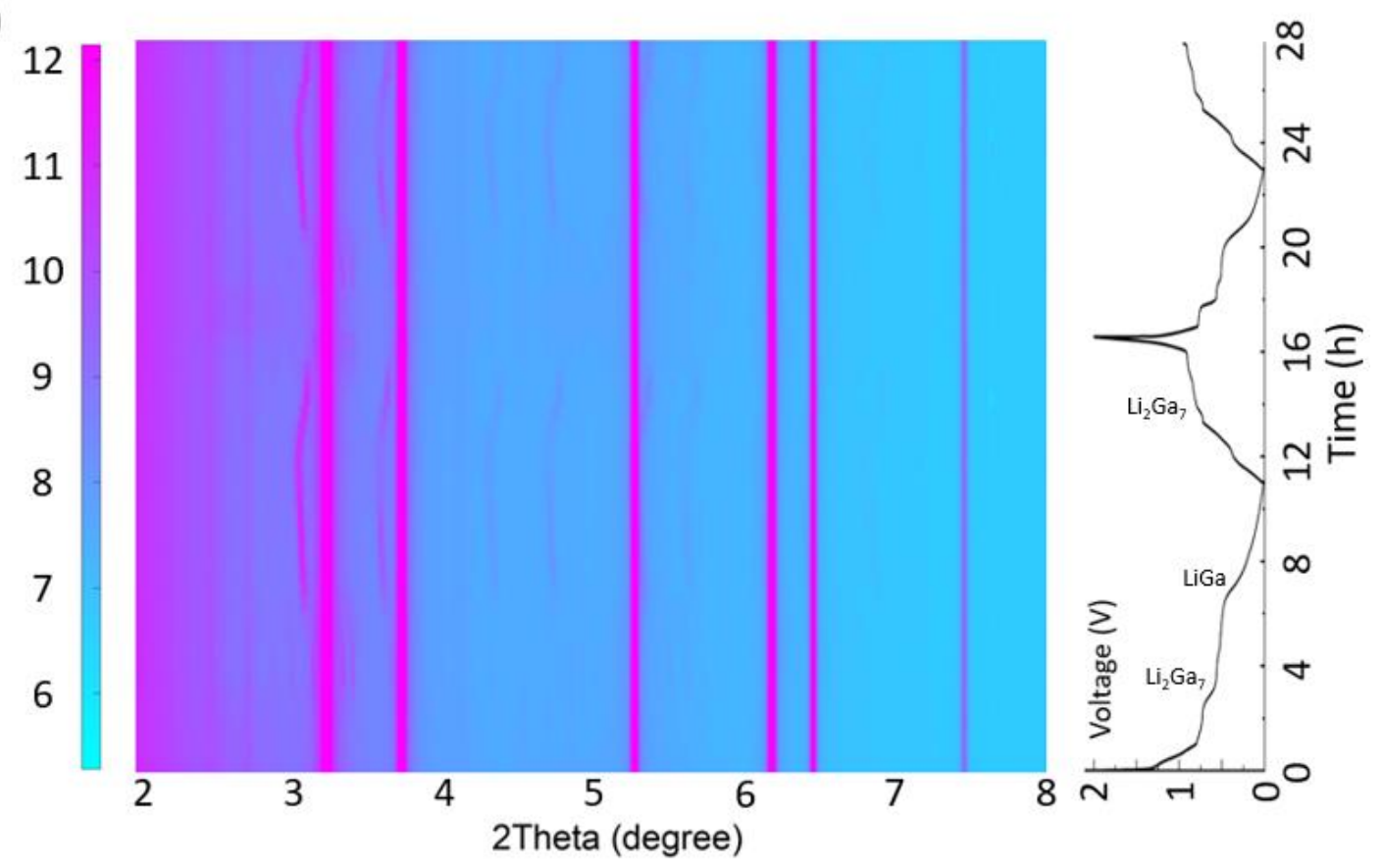

b)

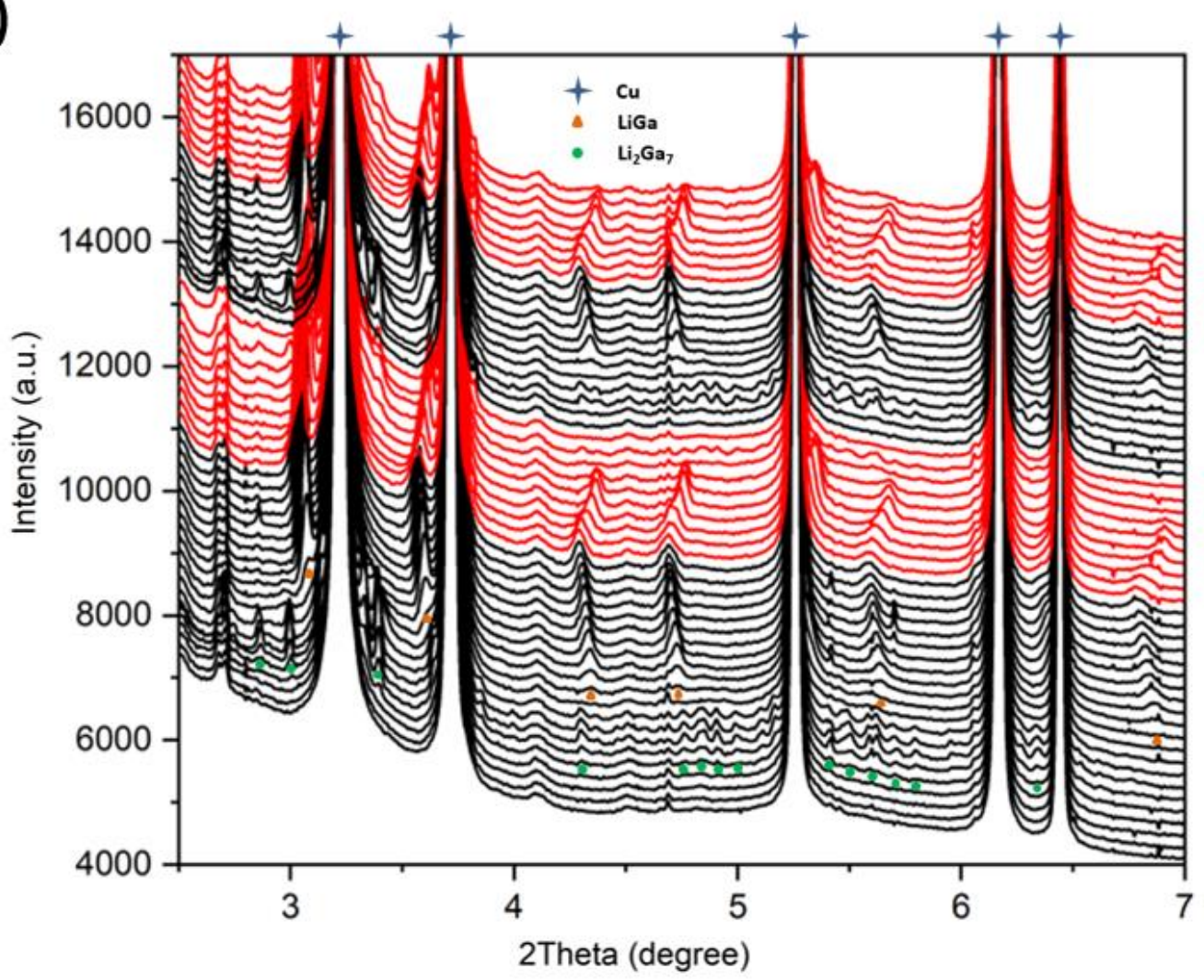

Figure 3. (a) The operando XRD results with potential plot and (b) elected XRD patterns by 32 min time step. 
The electrochemical behavior of the LMNPs with PEO solid electrolyte was evaluated by cyclic voltammetry $(\mathrm{CV})$ in lithium half cells operated under $60{ }^{\circ} \mathrm{C}$ swept at 0.02 $\mathrm{mV} \mathrm{s}^{-1}$, as shown in Figure 4a. In the cathodic scan from the open-circuit voltage $(\mathrm{OCV})$ to $0.005 \mathrm{~V}$, the first obvious difference is the lack of a peak at $1.2 \mathrm{~V}$ for SEI formation, which is different from the CV curve using the liquid electrolyte. The lithium insertion mainly occurs at 0.73 and $0.52 \mathrm{~V}$ for $\mathrm{Ga}$ with another three small peaks at $0.77,0.55$, and $0.35 \mathrm{~V}$ correspond to the gallium bond cleavage associated with the formation of $\mathrm{Li}_{2} \mathrm{Ga}_{7}, \mathrm{LiGa}$, and $\mathrm{Li}_{2} \mathrm{Ga}$, respectively. The anodic lithium extraction processes occur at $0.23,0.38,0.72,0.83$, and $0.89 \mathrm{~V}$ for the reverse process from $\mathrm{Li}_{2} \mathrm{Ga}$ to $\mathrm{LiGa}$ followed by $\mathrm{Li}_{2} \mathrm{Ga}_{7}$ and eventually back to Ga-Sn LM. The hightemperature CV result with PEO electrolyte shows Sn does not participate in any electrochemical reactions, which is consistent with the operando XRD experiment. We also conducted ex situ XRD to understand the phase changes of LMNPs in the solid electrolyte cell. As shown in Figure S5, only the $\mathrm{Li}_{2} \mathrm{Ga}$ phase appeared after full lithiation and no $\mathrm{Li}_{\mathrm{x}} \mathrm{Sn}$ phases were observed during cycling. The lithium ion diffusion rate is two orders of magnitude faster in Ga LM compared to that in Sn at 60 ${ }^{\circ} \mathrm{C}[29]$. Therefore, it is difficult for this small amount of $\mathrm{Sn}$ to form any $\mathrm{Li}_{\mathrm{x}} \mathrm{Sn}$ phases during cycling processes. As shown in Figure $\mathbf{4 b}$, a small dip was observed during the first discharge when the potential reaches $0.77 \mathrm{~V}$ which means activation energy was required for bond cleavage between $\mathrm{Ga}-\mathrm{Ga}$ bond and phase formation of $\mathrm{Li}_{\mathrm{x}} \mathrm{Ga}$ as reported in ref [39]. This small portion of Sn doping has the ability to reduce the melting point of Ga-Sn LMNPs which benefits the crystalline evolutions of the $\mathrm{Ga}$ 
phase. Compared with the previous study reported by Saint et al., the formation of the $\mathrm{Li}_{2} \mathrm{Ga}$ phase mainly occurs once the voltage reaches $0.01 \mathrm{~V}$ that needs more kinetic energy satisfied by either very low current or high temperature. Consequently, we are able to observe the formation of $\mathrm{Li}_{2} \mathrm{Ga}$ as shown in Figure $\mathbf{4 a}$ and $\mathbf{S 5}$ when we operate the cells at $60{ }^{\circ} \mathrm{C}$ but not for the operando experiments conducted at room temperature.
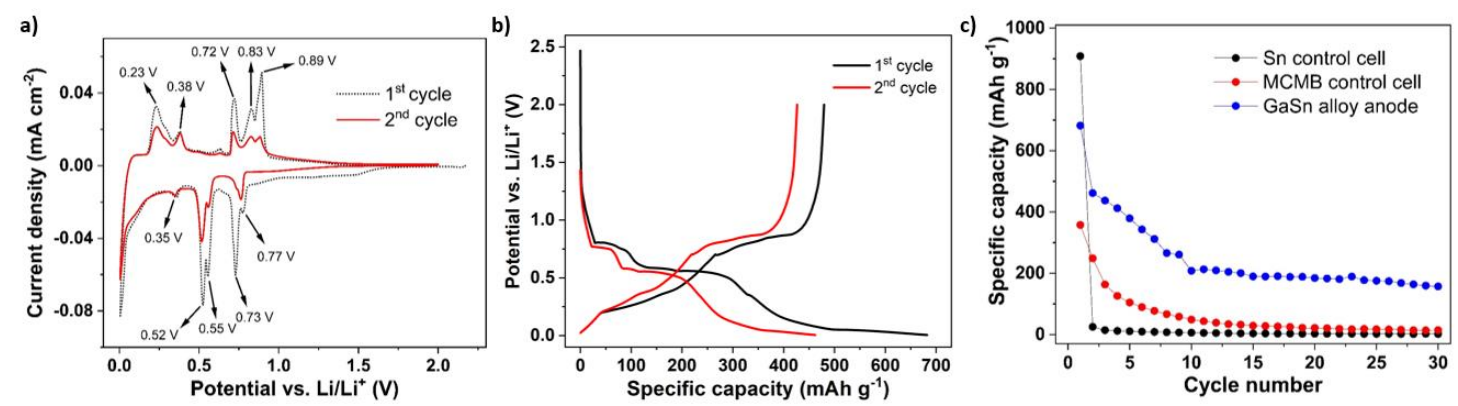

Figure 4. (a) Cyclic voltammetry of the Ga-Sn LMNPs electrode tested at $60{ }^{\circ} \mathrm{C}$. The potential was swept from open-circuit voltage to $0.005 \mathrm{~V}$ and then swept back to 2.0 $\mathrm{V}$ at a scanning rate of $0.02 \mathrm{mV} \mathrm{s}^{-1}$. (b) Potential capacity plots of the Ga-Sn LMNPs electrode tested at $60{ }^{\circ} \mathrm{C}$. The cell was galvanostatically cycled at $\mathrm{C} / 20$ rate in voltage ranges of 0.005-2.0 V. (c) Comparison of Ga-Sn LMNPs electrode with Sn and MCMB electrodes under the same operational conditions.

The cycling performance for LMNPs was evaluated by combining an LMNPs electrode with a PEO solid electrolyte to form a full solid half cell tested in an Espec environmental chamber maintained at $60{ }^{\circ} \mathrm{C}$ during cycling. The picture of the PEO solid electrolyte is shown in Figure S6 with the SEM image showing its morphology. 
To highlight the electrochemical advantages of the Ga-Sn LMNPs, its cycling test was compared to the Sn particle electrode (with large volume change) and MCMB electrode (without large volume change) under the same conditions respectively, as shown in Figure 4c. The selected voltage-capacity plots of Ga-Sn LMNPs, Sn particles, and MCMB electrodes are shown in Figure S7-9, respectively. The Ga-Sn LMNPs have an initial lithiation capacity of $682 \mathrm{mAh} \mathrm{g}^{-1}$ which is corresponding to approximately $87 \%$ of the theoretical capacity. After that, it fades to a lithiation capacity of $462 \mathrm{mAh} \mathrm{g}^{-1}$ at second lithiation and retains at $208 \mathrm{mAh} \mathrm{g}^{-1}$ in the first 10 cycles. Afterward, it continuously fades to $158 \mathrm{mAh} \mathrm{g}^{-1}$ after 30 cycles. There is a fast capacity drop (about 70\%) during the first 10 cycles. We believe that many Ga-Sn LMNPs lost contact with the PEO solid electrolyte and became inactive. Although the Ga-Sn electrode has the advantageous features of liquid, such as flexibility and fluidity, the large volume change upon cycling is still a big challenge for maintaining the contact between active materials and solid electrolytes. The cycling results also show that the capacity drop is mitigated after the first 10 cycles. The capacity drops from the $10^{\text {th }}$ cycle to the $30^{\text {th }}$ cycle is about $7 \%$ of its originally delivered. It indicates that Ga-Sn LMNPs have a possibility to maintain contact with PEO solid electrolytes with a future optimized microstructural design of the electrode. The Sn particle electrode was tested as a baseline. It has an initial lithiation capacity of $909 \mathrm{mAh} \mathrm{g}^{-1}$ and then rapidly fades to $25 \mathrm{mAh} \mathrm{g}^{-1}$ at the $2^{\text {nd }}$ cycle. The capacity drop is over $97 \%$. It is not surprising because of the large volume change of Sn during the first cycle deactivated most of the particles. MCMB was also tested as a baseline experiment, in 
which the MCMB electrode has an initial lithiation capacity of $358 \mathrm{mAh} \mathrm{g}^{-1}$.

Subsequently, it fades to $249 \mathrm{mAh} \mathrm{g}^{-1}$ at the $2^{\text {nd }}$ cycle with continuous capacity fading to $49 \mathrm{mAh} \mathrm{g}^{-1}$ in the first 10 cycles and eventually retains at $15 \mathrm{mAh} \mathrm{g}^{-1}$ after 30 cycles. The capacity drop is about $86 \%$ during the first 10 cycles and another $9 \%$ was lost from the $10^{\text {th }}$ cycle to the $30^{\text {th }}$ cycle. By comparing with $\mathrm{Sn}$ and MCMB electrodes, it is clear that the liquid metal electrode can maintain better contact with PEO solid electrolytes, mainly due to its advantageous features of the liquid.

However, the liquid metal undergoes a liquid-solid-liquid transition during cycling, which can lead to the gradual loss of contact upon cycling. This problem could be solved by several potential approaches, such as optimizing the microstructure of the electrode and maintaining larger pressure on the electrode.

\section{Conclusion}

In this study, we demonstrate the concept of using Ga-Sn LMNPs as low melting point anode materials for lithium ion batteries. The morphological evolutions were investigated via ex-situ SEM and operando TXM, which indicates the reversibility of nm-sized active materials during cycling. The Ga-Sn LMNPs demonstrate selfhealing ability at room temperature, which avoided the cracking and delamination issues caused by volume expansion during cycling. The operando and ex situ XRD results revealed that the crystalline evolution of the Ga-Sn electrode is Ga-Sn liquid metal $-\mathrm{Li}_{2} \mathrm{Ga}$ - $\mathrm{LiGa}-\mathrm{Li}_{2} \mathrm{Ga}$. There is a solid solution formation process when lithium is inserted into $\mathrm{LiGa}$ crystal. $\mathrm{Li}_{2} \mathrm{Ga}$ will be formed only with very low current 
or high temperatures. The $8 \%$ Sn was not observed to participate in the electrochemical reaction to form $\mathrm{Li}_{\mathrm{x}} \mathrm{Sn}$ alloys reversibly by both operando XRD and the CV test. By employing a PEO-based solid electrolyte at an elevated temperature of $60{ }^{\circ} \mathrm{C}$, the liquid metal anode showed reversible lithium insertion and extraction and a better cycling capability than the control cells with Sn and MCMB anodes. These results indicate that the liquid metal electrode can maintain the mechanical integrity of the electrode and better contact with PEO solid electrolytes, due to its advantageous features of liquid, such as self-healing ability and fluidity. This study offers a new strategy to employ a combination of liquid metal alloys and polymer solid electrolytes to address some challenges in rechargeable lithium ion batteries with solid electrolytes. 


\section{Acknowledgment}

This work was supported by US National Science Foundation under Grants No.

1603847. This research used resources of the Advanced Photon Source, a U.S.

Department of Energy (DOE) Office of Science User Facility, operated for the DOE

Office of Science by Argonne National Laboratory under Contract No. DE-AC02-

$06 \mathrm{CH} 11357$.

\section{Author Statements}

Tianyi Li: Methodology, Investigation, Formal analysis, Writing- Reviewing and

Editing. Yi Cui: Methodology, Investigation, Writing- Original draft preparation.

Longlong Fan: Formal analysis. Xinwei Zhou: Resources. Yang Ren: Resources.

Vincent De Andrade: Resources. Francesco De Carlo: Resources. Likun Zhu:

Conceptualization, Funding acquisition, Supervision 


\section{References:}

1. Zhang, W.-J., A review of the electrochemical performance of alloy anodes for lithium-ion batteries. Journal of Power Sources, 2011. 196(1): p. 13-24.

2. Lim, L.Y., et al., Understanding Phase Transformation in Crystalline Ge Anodes for LiIon Batteries. Chemistry of Materials, 2014. 26(12): p. 3739-3746.

3. Lim, L.Y., et al., Storage Capacity and Cycling Stability in Ge Anodes: Relationship of Anode Structure and Cycling Rate. Advanced Energy Materials, 2015. 5(15): p. 1500599.

4. Klavetter, K.C., et al., High tap density microparticles of selenium-doped germanium as a high efficiency, stable cycling lithium-ion battery anode material. Journal of Materials Chemistry A, 2015. 3(11): p. 5829-5834.

5. $L i, T$., et al., Characterization of dynamic morphological changes of tin anode electrode during (de)lithiation processes using in operando synchrotron transmission $X$-ray microscopy. Electrochimica Acta, 2019. 314: p. 212-218.

6. $\quad \mathrm{L}, \mathrm{T}$. , et al., In Situ and Operando Investigation of the Dynamic Morphological and Phase Changes of Selenium-doped Germanium Electrode during (De)Lithiation Processes. Journal of Materials Chemistry A, 2019.

7. Wang, J., K. Chen - Wiegart Yu - chen, and J. Wang, In Situ Three - Dimensional Synchrotron X - Ray Nanotomography of the (De)lithiation Processes in Tin Anodes. Angewandte Chemie International Edition, 2014. 53(17): p. 4460-4464.

8. Wang, J., et al., Visualization of anisotropic-isotropic phase transformation dynamics in battery electrode particles. Nature Communications, 2016. 7: p. 12372.

9. Zhou, X., et al., In Situ Focused Ion Beam-Scanning Electron Microscope Study of Crack and Nanopore Formation in Germanium Particle During (De)lithiation. ACS Applied Energy Materials, 2019.

10. Zhou, X., et al., In Situ Focused Ion Beam Scanning Electron Microscope Study of Microstructural Evolution of Single Tin Particle Anode for Li-lon Batteries. ACS Applied Materials \& Interfaces, 2019. 11(2): p. 1733-1738.

11. Iltis, G.C., et al., Imaging biofilm architecture within porous media using synchrotronbased X-ray computed microtomography. Water Resources Research, 2011. 47(2).

12. Müller, S., et al., Quantification and modeling of mechanical degradation in lithiumion batteries based on nanoscale imaging. Nature Communications, 2018. 9(1): $p$. 2340.

13. Chen, R., et al., Intermetallic SnSb nanodots embedded in carbon nanotubes reinforced nanofabric electrodes with high reversibility and rate capability for flexible Li-ion batteries. Nanoscale, 2019. 11(28): p. 13282-13288.

14. Gonzalez Malabet, H.J., et al., In Operando XANES Imaging of High Capacity Intermetallic Anodes for Lithium Ion Batteries. Journal of The Electrochemical Society, 2020. 167(4): p. 040523.

15. Nguyen, Q.H., et al., Enhanced performance of carbon-free intermetallic zinc titanium alloy (Zn-ZnxTiy) anode for lithium-ion batteries. Electrochimica Acta, 2019. 301: p. 229-239. 
16. Abel, P.R., et al., Nanostructured Si(1-x)Gex for Tunable Thin Film Lithium-Ion Battery Anodes. ACS Nano, 2013. 7(3): p. 2249-2257.

17. Liu, S., et al., Nanoporous germanium as high-capacity lithium-ion battery anode. Nano Energy, 2015. 13: p. 651-657.

18. Seng, K.H., et al., Self-Assembled Germanium/Carbon Nanostructures as High-Power Anode Material for the Lithium-Ion Battery. Angewandte Chemie International Edition, 2012. 51(23): p. 5657-5661.

19. Kennedy, T., M. Brandon, and K.M. Ryan, Advances in the Application of Silicon and Germanium Nanowires for High-Performance Lithium-Ion Batteries. Advanced Materials, 2016. 28(27): p. 5696-5704.

20. Park, M.-H., et al., Germanium Nanotubes Prepared by Using the Kirkendall Effect as Anodes for High-Rate Lithium Batteries. Angewandte Chemie International Edition, 2011. 50(41): p. 9647-9650.

21. Cho, Y.J., et al., Tetragonal Phase Germanium Nanocrystals in Lithium Ion Batteries. ACS Nano, 2013. 7(10): p. 9075-9084.

22. Liu, N., et al., A Yolk-Shell Design for Stabilized and Scalable Li-lon Battery Alloy Anodes. Nano Letters, 2012. 12(6): p. 3315-3321.

23. Wang, J., et al., Shell-Protective Secondary Silicon Nanostructures as PressureResistant High-Volumetric-Capacity Anodes for Lithium-Ion Batteries. Nano Letters, 2018. 18(11): p. 7060-7065.

24. $\mathrm{Wu}, \mathrm{H}$. and Y. Cui, Designing nanostructured Si anodes for high energy lithium ion batteries. Nano Today, 2012. 7(5): p. 414-429.

25. Wang, H., et al., A binder-free high silicon content flexible anode for Li-ion batteries. Energy \& Environmental Science, 2020. 13(3): p. 848-858.

26. Wu, Y., et al., A room-temperature liquid metal-based self-healing anode for lithiumion batteries with an ultra-long cycle life. Energy \& Environmental Science, 2017. 10(8): p. 1854-1861.

27. Guo, X., et al., A Self-Healing Room-Temperature Liquid-Metal Anode for Alkali-Ion Batteries. Advanced Functional Materials, 2018. 28(46): p. 1804649.

28. Ding, Y., et al., A Liquid-Metal-Enabled Versatile Organic Alkali-Ion Battery. Advanced Materials, 2019. 31(11): p. 1806956.

29. Deshpande, R.D., et al., Liquid Metal Alloys as Self-Healing Negative Electrodes for Lithium Ion Batteries. Journal of The Electrochemical Society, 2011. 158(8): p. A845.

30. Verbrugge, M.W., et al., The search for high cycle life, high capacity, self healing negative electrodes for lithium ion batteries and a potential solution based on lithiated gallium. MRS Proceedings, 2011. 1333: p. mrss11-1333-m05-04.

31. Liang, W., et al., Nanovoid Formation and Annihilation in Gallium Nanodroplets under Lithiation-Delithiation Cycling. Nano Letters, 2013. 13(11): p. 5212-5217.

32. Guo, X., et al., Room-temperature liquid metal and alloy systems for energy storage applications. Energy \& Environmental Science, 2019. 12(9): p. 2605-2619.

33. Jin, Y., et al., High-Energy-Density Solid-Electrolyte-Based Liquid Li-S and Li-Se Batteries. Joule, 2020. 4(1): p. 262-274. 
34. Jin, Y., et al., An intermediate temperature garnet-type solid electrolyte-based molten lithium battery for grid energy storage. Nature Energy, 2018. 3(9): p. 732738.

35. Getsoian, A.B., et al., Organometallic model complexes elucidate the active gallium species in alkane dehydrogenation catalysts based on ligand effects in Ga K-edge XANES. Catalysis Science \& Technology, 2016. 6(16): p. 6339-6353.

36. Sun, C., et al., Self-supported GaN nanowires with cation-defects, lattice distortion, and abundant active sites for high-rate lithium-ion storage. Nano Energy, 2020. 68 : p. 104376.

37. Bunker, G. The EXAFS of Disordered Systems and the Cumulant Expansion. in EXAFS and Near Edge Structure III. 1984. Berlin, Heidelberg: Springer Berlin Heidelberg.

38. Saint, J., et al., Exploring the Li-Ga room temperature phase diagram and the electrochemical performances of the LixGay alloys vs. Li. Solid State Ionics, 2005. 176(1): p. 189-197.

39. Lee, K.T., et al., Role of Electrochemically Driven Cu Nanograins in CuGa2 Electrode. Chemistry of Materials, 2008. 20(2): p. 447-453. 